\title{
Return to Athletic Activity Following Total Hip Arthroplasty
}

\author{
Jeremy Ross and Thomas E. Brown*
}

Depatment of Orthopedic Surgery, University of Virginia, Charlottesville, Virginia, USA

\begin{abstract}
As demand for total hip arthroplasty increases, patients are undergoing procedures at younger ages and often choose to maintain athletic activity after the operation. However, there is little evidence as to what level of athletics is acceptable post-operatively. To this point, no prospective studies have been performed to evaluate implant longevity at mid-term and long-term endpoints when patients return to athletics. Expert recommendations are available from the Hip Society and the American Association of Hip and Knee Surgeons. These suggestions can be used in conjunction with a thorough pre-operative evaluation to provide guidance for patient activity post-operatively. Surgeons should also consider anatomic and biomechanical factors along with surgical technique when providing patients with advice to prolong the life of their implant.
\end{abstract}

Keywords: Total hip arthroplasty, athletics, post-operative.

Since its acceptance into common orthopaedic practice in the 1970s, total hip arthroplasty (THA) has become a hallmark procedure in the field of orthopaedics. As the general population ages and continues to be highly active for many more years than previously, the rate of THA is expected to increase $174 \%$ from 209,000 procedures to 572,000 procedures per year in the USA [1]. Other contributions to this rise include an increasing prevalence of osteoarthritis, trauma, and obesity [2].

Patients who become candidates for THA commonly present with groin pain, lateral hip pain upon weight-bearing and restricted range of motion due to pain. Physical examination and radiographic imaging illustrate the intraarticular processes responsible for these complaints. When patients are unable to obtain symptom control with medical management and have significant impairment of daily activities due to pain, THA is indicated for symptom relief.

Modern surgical techniques and prosthetic implants have led to improved outcomes of THA. Thus, more patients are electing to undergo the procedure in order to improve their quality of life. As the population ages and remains more active than in the past, surgeons performing THA are often faced with the task of making recommendations for return to athletic activity post-operatively.

The topic of acceptable activities and sports participation following THA has led to many recommendations from orthopaedic surgeons, but a consensus on this debate has yet to be reached. Many groups and individuals have set forth guidelines for individual activities, and surgeons can use these to guide them in their practice. As the indications for THA continue to evolve and include younger patients and more active aging patients, these guidelines have allowed for motivated patients to return to an increasing number of

*Address correspondence to this author at the Depatment of Orthopedic Surgery, University of Virginia, Box 800159 HSC UVA, Charlottesville, VA 22908, USA; Tel: 434-243-0278; Fax: 434-2443-0290;

E-mail: tb4g@virginia.edu athletic endeavors. To date there are no long-term studies on the durability of THA components in patients returning to physical activity, but evidence is available that may help orthopaedic surgeons to make practical recommendations to highly motivated THA patients for post-operative activity.

\section{ETIOLOGY OF HIP PAIN}

The degenerative processes leading to hip pain can be divided into two categories based on their characteristic patterns of cartilage destruction [3]. Primary osteoarthritis of the hip is the most common process leading to THA, yet remains a diagnosis of exclusion after ruling out structural and metabolic pathologies. Secondary osteoarthritis of the hip refers to any process that causes pathologic destruction of the hip articular surfaces. This can include rheumatoid arthritis, septic arthritis, developmental dysplasia of the hip, Legg-Calvé-Perthes disease, and many others.

Secondary arthritis of the hip can be further divided into anatomic and pathologic categories. Examples of anatomic alterations that can lead to hip arthritis are slipped capital femoral epiphysis, developmental dysplasia of the hip, LeggCalvé-Perthes disease and other processes that lead to osteonecrosis of the joint. These anatomic changes ultimately lead to overloading of the native hyaline cartilage and over time failure of the cartilage. Pathologic insults to the joint include rheumatoid arthritis, septic arthritis, traumatic arthritis, and seronegative spondyloarthropathies. In these disease states, destruction of the hyaline cartilage occurs directly. Ultimately, both anatomic and pathologic processes lead to failure of the hyaline cartilage and eventually to the typical presenting symptoms of degenerative hip disease.

As stated previously, primary osteoarthritis of the hip becomes a diagnosis of exclusion and is used only when all secondary causes of osteoarthritis have been exhausted. Since there are no clear disease states in primary osteoarthritis, studies on the etiology of this process are epidemiological and seek to identify commonalities between patients. Epidemiologic considerations have led to the 
implication of a strong genetic component to the development of primary osteoarthritis. One study showed that American Caucasians of Western European descent carry a $3-6 \%$ rate of primary osteoarthritis. This rate is up to 10 times higher than African-Americans and up to 27 times higher than Asian Americans [4]. These rates are especially significant, considering that these groups have similar rates of secondary osteoarthritis. Also, it has been illustrated that relatives of patients who have undergone THA have findings suggestive of coxarthrosis in $27 \%$ to $50 \%[5,6]$. When considered together, there is a suggestion that the population of patients undergoing THA may have an underlying cartilage defect with a genetic contribution.

Other factors can contribute to the development of primary osteoarthritis. Studies have suggested that high body weight or BMI, heavy labor, elite sports participation, and femoral anteversion may additionally contribute to hip arthritis in patients predisposed to the condition. In fact, Swedish studies have shown that there is an increased risk for development of osteoarthritis in patients participating in track and field, racket sports like tennis, and soccer. Professional soccer players were shown to have the greatest risk for development of osteoarthritis of all patients in these studies [7].

\section{PRE-OPERATIVE CONSIDERATIONS}

At present, there is no widely accepted classification system for hip osteoarthritis. Experience has shown that correlation between radiographic findings of hip arthritis and clinical presentation is rare. Ultimately, patient perception of the impact of hip pain on their daily life after failing all medical management has been the indication for THA.

The most common presenting symptom in patients ultimately requiring THA is groin pain. The pain has typically worsened over time and has periods of improvement along with periods of worsening. Osteoarthritic hip pain usually increases with activity and is often worse as the day progresses. Also, higher load or impact activities worsen the pain. In some instances, the patient has had some recent mild trauma that leads to a notable increase in pain. If the pain occurs with weight-bearing and is alleviated by rest, this is a strong indication of intra-articular pathology. Morning stiffness typically lasts for less than one hour, and prolonged rest throughout the day can increase stiffness. In addition to groin pain, patients may present with lateral leg pain or pain in the area of the greater trochanter representing abductor tendinitis and trochanteric bursitis, respectively. These pathologies may be linked to hip osteoarthritis as pain leads to changes in gait and non-physiologic function of hip muscles and bony anatomy. Medial knee pain may be a complaint, and sometimes no hip pain is noted at all. In these atypical presentations, a thorough examination and hip radiographs should be obtained to examine possible hip pathology. A final common presentation is pain in the lower lumbar or gluteal area. Hip pain is rarely referred to these areas, and this complaint requires a complete evaluation for lumbar spine and sacroiliac pathology [8].

By the time patients present to an orthopaedic surgeon, they will have usually decreased their activity level significantly due to pain. Initially, they may decrease their participation in athletics. This typically progresses to avoidance of prolonged standing or ambulation. The pain can be so severe that patients are severely limited in their day-to-day activities and therefore have reached a point where they are considering operative intervention.

As with any patient encounter, a thorough physical exam is necessary. Several aspects that should be considered are weight-bearing status, range of motion, muscular strength, vascular status, and leg length discrepancy. In addition, there are myriad provocative tests that can be performed to further clarify pain. These maneuvers can help to rule out confounding pathology such as lumbar spine disease. Radiographic examination is helpful to corroborate the clinical history and physical exam findings. Standard films include an anteroposterior view showing as much of the pelvis as possible and a frog-leg lateral view. Obturator and iliac oblique views can illustrate the bony stock of the anterior and posterior columns. In patients who present with clinical evidence of intra-articular disease but show no radiographic evidence of degenerative changes, MRI is indicated to rule out avascular necrosis.

There are times when a thorough history and physical exam with provocative testing do not reveal a clear source of hip pain. This can often occur in patients with arthritis of many joints. In these cases, fluoroscopically guided injection of anesthetic with clinical exam before and after the intervention can be useful. If symptoms improve after anesthetic injection into the hip joint, then hip arthritis is diagnosed. This can also be useful for patients with concurrent hip and lumbar spine pain by illustrating what proportion of a patient's pain can be attributed to hip pathology. The ultimate goal of examination is to offer the patient an operation that will provide them with the most benefit in pain reduction.

Any patient presenting with unilateral hip pain suggestive of an intra-articular process deserves a trial of medical management before THA is pursued. However, most patients have exhausted such options by the time they are referred to an orthopaedic surgeon. The surgeon should feel obligated to ensure that all nonoperative measures have been tried before offering a surgical intervention. Medical management is generally regarded as having two categories: pharmacologic and nonpharmacologic treatments. A summary of medical management of hip pathology can be seen in Table $\mathbf{1}$.

Recent interest has been given to the specific modalities of physical therapy utilized prior to THA. Many surgeons anecdotally recommend pool-based therapy over land-based therapy as being lower impact and therefore leading to less pain. A study by Gill et al. looked into this assertion by comparing pain and self-reported functional status in patients awaiting total hip or knee replacement who underwent either land-based or pool-based therapy. They found that both interventions were effective at improving pain and increasing function, but there were no significant posttherapy differences in functional status between the two groups. However, it was noted that pool-based exercise led to less pain immediately following therapy [9]. Patients can therefore be advised that physical therapy is effective with either pool- or land-based modalities.

When medical management has been exhausted and patients continue to have debilitating hip pain, total hip 
arthroplasty is typically pursued. Circumstances do arise where hip arthroscopy may be considered, but these are limited in patients with established arthritis. Patients in whom arthroscopy can be beneficial include athletes with minimal degenerative changes on radiographic exam but with soft tissue findings on MRI like a labral tear or cartilage flap. When considering THA for current athletes or active individuals wishing to return to sports, the durability and stability of the implant is paramount. The patient and surgeon should have a frank discussion regarding realistic expectations of return to activity pre-operatively as this typically improves patient satisfaction with the operative correction [10]. Attention should also be brought to how the patient's activities will impact the endurance of the implant. Careful analysis of pre-operative activity level, patient desire to return to activity, type of fixation, and bearing type can lead to quality outcomes in active patients wishing to return to sports following THA.

\section{Table 1. Medical Management of Hip Arthritis}

Physical Therapy
Range of motion exercises
Muscular strengthening exercises
Ambulation assistive devices
Occupational Therapy
Joint loading protection
Assistive devices for activities of daily living
Weight loss
Patient Education
Aquatic aerobic exercise programs
Pharmacotherapy
Nonopioid analgesics (acetaminophen)
Nonsteroidal anti-inflammatory drugs
Opioid analgesics
Intra-articular anesthetic/steroid injection

\section{ANATOMIC AND BIOMECHANICAL CONSIDER- ATIONS}

When the anatomic and biomechanical aspects of hip joint function are considered, a proper implant may be chosen for the patient who wishes to return to sporting activity following THA. As expected, stability should be the principal deciding factor of operative success as sports participation places the implant at higher risk of dislocation. However, proper pre-operative planning and counseling can lead to a successful return to activity following THA.

The proximal femoral osteology pertinent to hip joint pathology includes the head, neck, greater and lesser trochanters, and proximal femoral diaphysis. The femoral head averages $46 \mathrm{~mm}$ in diameter and the average neck-shaft angle is $125 \pm 7$ degrees [11]. Anteversion of the femur is determined by the angle formed between the femoral epicondyles and the plane of the femoral neck and averages $13 \pm 7$ degrees in normal patients. However, the average anteversion in patients with coxarthrosis of the hip is $20 \pm 9$ degrees [12]. The proximal femur has a posterior bow which intersects the anterior bow of the femoral shaft at the lesser trochanter. The posterior bow of the proximal femur must be considered for proper placement of the femoral stem in the sagittal plane during THA.

The acetabulum is formed by the fusion of the ilium, ischium, and pubis. The dome of the acetabulum provides the most support of the femoral head by giving direct cephalad coverage. The thin medial wall of the acetabulum provides only minimal support. The densest bone of the acetabulum is located in the posterior and superior area [13]. The acetabulum is anteverted at an average of $17 \pm 6$ degrees and faces caudally. Acetabular anteversion and inclination must be considered during THA as an average of 15 degrees of anteversion and 35-45 degrees of inclination of the acetabular component are required.

The muscular anatomy about the hip is an important consideration during THA. The two most common surgical approaches to the hip in practice today are the anterolateral and posterior approaches. It is for this reason that the medial thigh muscles are not usually encountered during THA. All posterior approaches to the hip capsule require taking down the short external rotators while maintaining the abductors. In contrast, anterolateral and lateral approaches transect a portion of the gluteus medius and minimus to reach the hip capsule. With lateral approaches, care must be taken to ensure sturdy reattachment of the gluteus muscles to ensure post-operative stability of the joint.

The biomechanical functions to be considered during THA are motion, stability, and force transmission across the hip joint. An average patient without hip arthritis will lose about 0.5 to 1 degree of range of motion per decade after skeletal maturity has been reached. It has been previously illustrated that average patients aged 40-59 have 120 degrees of flexion, 18 degrees of extension, 42 degrees of abduction, 31 degrees of internal rotation, and 32 degrees of external rotation when no arthritis is present [14]. Thus, the primary goal of THA should be to restore as much of this range of motion as possible. The concept of head-to-neck ratio becomes important for range of motion and stability after THA. Small femoral prosthetic heads have a decreased range of motion as compared to larger prostheses. This occurs as the smaller heads lead to earlier impingement of the femoral neck against the acetabular component, thereby limiting range of motion. However, a small femoral head component can have an adequate range of motion by decreasing femoral neck size. It is the ratio of head size to neck size that determines the range of motion. Constrained femoral head prostheses can have as little as 70 degrees range of motion, but may be necessary in patients who chronically dislocate prosthetic hips. Newer designs of constrained components can allow a nearly normal range of motion. There has been recent interest in total hip resurfacing and metal-on-metal prostheses as these options allow the use of a very large femoral head. These maximize the head-to-neck ratio and allow an excellent range of motion.

The native hip has a great deal of inherent stability, and successful THA requires that post-operative stability of the joint be maximized, especially if a return to athletics is desired. A normal hip has many constraints to dislocation and instability provided by the bony architecture, strong capsule, and overlying ligaments. Past experiences with instability of implants has led to awareness of prosthesis design, implant orientation, surgical technique, and soft 
tissue tensioning as critical constituents of stability. If anatomic orientation of implants occurs, proper soft tissue tension should be preserved. Thus, there are two manners in which to increase post-operative hip joint stability. The first is to increase the size of the femoral head as discussed previously. In addition to increasing range of motion before impingement, large femoral heads have an increased "jump height" or distance the head itself must travel out of the acetabulum to dislocate. Another method involves the use of constrained femoral head prostheses, which provide more complete coverage of the femoral head to decrease dislocation while also decreasing range of motion [15]. When constrained systems are subject to impingement, however, they undergo tremendous torque stress and are subject to malfunction.

Properly positioning implants in anatomic orientation leads to improved soft tissue tension. This increases the stability of the post-operative hip and impacts load transmission across the hip joint. Forces across the hip can be up to 3 times body weight during walking and up to 8 times body weight while catching oneself from a stumble [16]. It is therefore vital that force transmission across the hip be optimized. For this reason, high-offset femoral stems have come into use in THA. The high-offset femoral stem has a decreased neck-shaft angle with a lengthened neck [17]. This combination allows the surgeon to effectively move the greater trochanter further lateral from the pelvis. Mechanically, this gives the abductor muscles a longer lever arm to act against and requires less force generation by the muscles to generate an equivalent force about the hip joint. Increasing the femoral offset also lengthens the abductor muscles, thus inherently increasing their contraction efficiency. By making the abductors more efficient in their contraction, force transmission across the hip joint is lessened.

\section{ISSUES WITH SURGICAL APPROACH}

Analysis of currently available evidence and recommendations reveals that several factors should be considered when discussing return to sports with postoperative THA patients. The surgical approach used and durable fixation of the prosthesis are the primary determinants to success. Also, the bearing type and activity level must be considered with their relationships to prosthetic wear and longevity of implants. Finally, stratification of activity levels can be useful to help determine the expected durability of implants in the face of varying demands on the bearing couple.

The choice of surgical approach for THA can have great implications on returning to sports in active patients. As the posterior, lateral, and anterolateral approaches are the most commonly used today, it is important to consider their advantages and shortcomings. Utilizing the posterior approach spares the abductor muscles, but requires taking down the short external rotators and the posterior capsule. In the past, this method has been associated with a dislocation rate of up to $5.8 \%$ as shown in one large study [18]. More current data have yielded different results and show that the rate of dislocation with the posterior approach is nearly equal to that of other approaches when the posterior capsule is not split through the posterior transverse aspect and when it is used along with the short external rotators to create a more complete deep closure. Two studies have shown dislocation rates of $0.7 \%$ and $0.8 \%[19,20]$ using this method of closure. The main shortcoming of the posterior approach is that it yields a higher risk of damage to the sciatic nerve. The major benefit of the posterior approach is the sparing of the abductor muscles. This technique is very important to stability and reestablishment of normal hip biomechanics following surgery.

The lateral and anterolateral approaches have historically yielded lower rates of dislocation as the operation can be performed without violation of the short external rotators and posterior capsule. This technique may offer more direct acetabular visualization and therefore potentially easier component placement. Studies of the dislocation rate using the lateral or anterolateral approach have yielded rates of $0.55 \%$ and $2.18 \%$ respectively [21]. The major drawback of these approaches is the violation of the abductors, and patients may be left with abductor weakness and a Trendelenburg gait. The study mentioned above showed a 4$20 \%$ limp rate using the lateral or anterolateral approach compared to $0-16 \%$ limp rate using the posterior approach to the hip joint.

A short-term outcome based study has been published which compares posterior and lateral approaches for THA. In this prospective, randomized trial, the Harris hip score was evaluated pre-operatively and at one, four, six, and twelve weeks post-operatively. From this data, the authors showed a slight tendency for Harris hip scores to be better at twelve weeks following THA via the posterior approach [22]. However, the authors caution that the slight improvement in Harris hip scores that they noted may not be clinically relevant. Therefore, conclusive data does not yet exist illustrating a clear functional advantage of one approach over the other.

The recent popularity of minimally invasive or minimal incision surgery among patients and surgeons has led to many studies comparing its use to traditional approaches. Thus far, data have only shown minimally invasive surgeries to improve patient recovery in the immediate post-operative period. Short-term and mid-term outcome data show no advantage for patients undergoing minimally invasive hip surgery. There are no long-term studies on this methodology to date. There are reports of component misalignment and fracture as complications of these approaches. Berger's two incision approach has gained popularity and is reported to provide better visualization than previous attempts at minimally invasive hip surgery [23]. Also, there are many modifications of traditional approaches that have been developed recently. The "anterior supine intermuscular" approach uses only the distal portion of the Smith-Peterson anterior approach with the proposed advantage of needing no transection of muscle or tendon to gain access to the hip joint.

\section{IMPLANT AND FIXATION CHOICES}

Durable fixation of implants is necessary for all arthroplasties, but requires extra consideration for the patient hoping to return to sports. Historically, cemented acetabular components showed good results early in their use. They became prone to loosening at an intolerably high rate after 
just a decade in spite of improved cementing techniques [24]. It was these poor results that led to the development of the porous coated acetabular components used today. The porous coating allows for bony ingrowth of the component, and current generation devices have an exceptional longevity rate of greater than $90 \%$ at more than 10 years follow-up [25].

Fixation of the femoral stem has been a bit more controversial than acetabular fixation owing largely to a lack of discrepancy in outcomes between press-fit and cemented stems. The Charnley flat-back polished femoral stem has long-term follow-up data showing a revision rate of $3 \%$ at 30 years [26]. However, when used in patients younger than 30 years old, this implant had revision rates of up to $10 \%$ at 30 years. Additional studies have corroborated this evidence and illustrated decreased cemented femoral stem component longevity in younger and more active patients [27]. Anecdotally, some surgeons recommend cemented femoral stems for older patients who are less active and have osteopenic bone.

The bearing couple must also be given careful consideration for more active THA patients. Currently, there are four bearing couples approved for use in the United States by the U.S. Food and Drug Administration: metal-onpolyethylene, ceramic-on-polyethylene, ceramic-on-ceramic, and metal-on-metal. In addition, metal-on-ceramic and diamond surfaces are under investigation. The rate of wear and quantity of debris produced are of primary importance in the choice of bearing couple. Two different categories of bearing couple can be considered: hard-on-soft, for example metal-on- polyethylene, and hard-on-hard, like metal-onmetal.

Metal- or ceramic-on-polyethylene comprise the hard-onsoft bearing couples. The most commonly used metal femoral head in the United States is cobalt-chromium alloy. The metal-on-polyethylene bearing couple is the most extensively employed and thus has the most wear data available. Cobalt-chromium on highly cross-linked polyethylene has an initial wear of 100 microns and a steady state wear of 10-20 microns per year. Data is inconsistent about the possibility that ceramic-on-polyethylene decreases the wear of the polyethylene surface. A ceramic head theoretically could yield less wear of the polyethylene liner as its surface hardness makes it less prone to the scratches and other damage taken by cobalt-chromium heads.

Experience with metal-on-metal bearing couples has been historically poor. However, recent studies have shown some metal on metal implants to be functioning well at 2030 years follow-up. Improvements in manufacturing techniques and tolerances have led to much better wear data with the metal-on-metal bearing and have led to resurgence in its popularity. The wear rate of metal on metal has been found to be 5-10 times less than hard on soft bearing couples. The initial wear of a metal on metal articulation is 25 microns and the steady state wear is around 5 microns per year. This decreased wear rate is not without consequences. There is much concern over the biologically active ions formed during wear of metal-on-metal bearings. The number of particles generated is 100-200 times greater than the number formed by hard-on-soft bearings. These ions have been shown to be toxic to cells and can induce chromosomal changes, so carcinogenicity is concerning. To date, no causal relationship has been discovered. Also, metal-on-metal bearings have been reported to squeak and click over time, but the incidence of this has been reduced owing to modern manufacturing techniques. Seizing of metal-on-metal implants has been historically reported, but again modern manufacturing has nearly eliminated this due to improved lubrication traits. The larger head size of modern components leads to greater surface area and higher angular or sliding velocities within the joint, thus maintaining the fluid film [28].

As compared to all other bearing couples, ceramic-onceramic bearings have the best wear rate. Initially, alumina ceramic bearings were used and had poor results due to the brittle nature of alumina. The current generation of ceramic implants has incorporated both the original alumina with zirconia to produce a material with the maximal toughness and fracture resistance of zirconia, along with the wear characteristics of alumina. Changes in the design and manufacture of ceramic components have also improved their durability [29]. If the femoral neck is allowed to impinge upon the ceramic acetabular liner, fracture of the liner is possible. Thus, designs protective of the liner and attention to intra-operative implant positioning are critical to prevent this outcome. The average initial wear of ceramicon-ceramic bearings is 1 micron with steady state wear of 0 3 microns per year. This wear rate is 3-5 times lower than metal-on-metal bearings. The quantity of wear particles generated is similar to metal-on-metal couples, but the particles produced seem to be less biologically active.

In recent years, hip resurfacing procedures have gained popularity among surgeons performing THA. One reason for this popularity is the preservation of native femoral bone that is possible with resurfacing compared to THA. Also, the ability to use a large femoral head component is appealing for stability and prevention of dislocation. By preserving the native femoral neck, it is thought that load transmission across the hip joint may mimic normal physiologic conditions and could therefore decrease stress on the acetabular component. Also, less stress shielding of the proximal femur should occur with hip resurfacing than in a totally replaced hip joint.

Since the conception of hip resurfacing arthroplasty nearly 50 years ago, there have been many changes in prosthetic materials used. Early designs utilized a metal acetabular component along with a metal femoral component which had high fail rates, similar to early metal-on-metal THA implants. Over time, this concept evolved to using a metal femoral head prosthesis along with cementing a polyethylene liner into the acetabulum. However, this combination led to very high wear rates and remarkable osteolytic reactions. Currently, the use of a thin metal acetabular component with porous ingrowth surfaces has increased the longevity and wear of hip resurfacing implants.

As with any arthroplasty, hip resurfacing comes with its own constraints. The procedure inherently allows a maximum of $1 \mathrm{~cm}$ of leg lengthening and no alteration of the femoral offset [30]. There are very limited and implant specific methods of augmenting acetabular fixation with screw placement. Also, significant bony loss that would prevent a stable press-fit is a contraindication to use. Bony 
loss in the femoral head from any pathologic state can compromise cement fixation of the femoral implant and therefore decrease its durability. Relative contraindications to the procedure include body mass index over 35, age greater than 60 years, female gender, and tall patients. A study with 5-years follow-up after fixation using a press-fit acetabular component with a cemented femoral component showed 96\% survivorship [31]. Femoral neck notching and varus placement of the femoral component can increase risk of femoral neck fracture, thereby decreasing the durability of the implant.

There is little data available on patient outcomes following hip resurfacing arthroplasty. Two retrospective studies have been performed via postal surveys that report on patient return to sports following the procedure. The first study involved 43 patients who had undergone 51 hip resurfacing procedures. Pre-operatively, $65 \%$ of patients participated in sports activities, while post-operatively the number increased to $92 \%$ [32]. The authors thus came to the conclusion that hip resurfacing allows patients to return to athletic activities that they were unable to participate in previously. A second study showed that 110 of 112 patients undergoing hip resurfacing $(98 \%)$ were able to return to multiple sports [33]. Of these patients, 51\% returned to downhill skiing, $12 \%$ to high-impact sports, and $22 \%$ to contact sports. The authors determined that hip resurfacing permitted patients to return to a high level of activity, but admitted that long-term wear and revision rates were needed. Thus, there are no current studies which validate the premise that hip resurfacing allows patients to safely return to highimpact sporting activities. This assertion is supported by Wylde and colleagues who showed no increased rate of return to sports following hip resurfacing arthroplasty when compared to THA [34]. Thus, patients hoping for a full return to activity following hip resurfacing should be duly counseled on the available outcome data.

\section{EVIDENCE BASIS FOR RETURN TO SPORTS FOLLOWING THA}

To date, there are no prospective controlled studies on longevity of THA implants in patients returning to athletic activities. There are myriad recommendations as to what activities are acceptable and at what level of performance they can be done. Evolution of these recommendations has naturally occurred over the past three decades. An early study by Visuri and Honkanen described the spontaneous return to recreational activity following THA [35]. In this study, 539 patients were identified from the Swedish Registry. The average patient age was 64 years and the mean follow-up was 4.2 years post-operatively. Regular walking increased from $2 \%$ pre-operatively to $55 \%$ post-operatively. Cycling increased from $7 \%$ to $29 \%$, swimming from $13 \%$ to $30 \%$, and skiing from $0 \%$ to $9 \%$. It was concluded from this study that swimming and cycling were valuable postoperative sporting activities because they produced the lowest joint stress. A 1983 study from Europe by Dubs and associates looked at 110 male patients with an average age of 55 years [36]. This analysis showed a contradictory rate of femoral stem component loosening of $14.3 \%$ in sedentary patients compared to just $1.6 \%$ in patients participating in sports post-operatively. In the patients returning to athletics, however, the acetabular liner wear rate was four times higher than in the sedentary group. Before this early data was available, surgeons placed severe restrictions on athletic activity following THA. The authors of the study deemed this unnecessary, but made the recommendation of using viscoelastic heel inserts to decrease peak joint loading during sporting activity.

A 1987 study of pre-operative and post-operative patient activity level surrounding THA was performed by Ritter and Meding [37]. It was found that post-operatively, all patients had a decreased level of participation in many athletic activities, except for cycling. Most patients returned to sports, but at intensity lower than they previously attained. It was concluded that low-impact activities like golf, walking, and bowling would have no impact on the longevity of THA implants. A report in 1991 showed twice the revision rate for patients who were highly active after THA or total hip resurfacing [38]. The decreased longevity became apparent after just 10 years in patients with osteoarthritis. Patients who underwent arthroplasty for other pathologies such as avascular necrosis had worse outcomes with signs of failure as early as 6 years post-operatively. Patients undergoing hip resurfacing experienced early loosening if they were involved in high-impact sports. A 2005 report by Huch and colleagues looked at 636 patients at 5 years after total joint replacement, either hip or knee [39]. Pre-operatively, 36\% of patients undergoing THA participated in athletics. Postoperatively, this number climbed to $52 \%$, which was higher than the post-operative data for total knee replacement (TKR). The authors suggest that the drastic improvement seen in THA patients when compared to TKR is due to greater pain relief in the THA group.

The first survey of orthopaedic surgeons regarding their recommendations on returning to sports following arthroplasty was performed in 1995 at the Mayo Clinic [40]. The questionnaire was distributed to attending physicians, fellows, and senior residents, and sought opinions on 28 common sports. From this, activities recommended following total hip or knee arthroplasty included sailing, lap swimming, scuba diving, cycling, golfing, and bowling. Sports discouraged were running, water-skiing, American football, baseball, basketball, hockey, handball, karate, soccer, and racquetball. The recommended activities were largely low-impact while the restricted activities were mostly high-impact. These data also provided the first categorization of sports into recommended, intermediate, and not recommended categories based on surgeon approval. A similar study was performed in 2001 and included recommendations by members of the Hip Society, Knee Society, and Elbow Surgeons Society regarding their opinions on 42 sports [41]. This survey categorized sports into allowed, allowed with experience, or not recommended. Additionally, the category of no conclusion was included for sports that did not have a consensus opinion. From the survey, the Hip Society placed the following sports into the allowed category: stationary cycling, croquet, ballroom dancing, golf, horseshoes, shooting, shuffleboard, swimming, doubles tennis, and walking. Sports that were allowed with experience were low-impact aerobics, road cycling, bowling, canoeing, hiking, horseback riding, and cross-country skiing. At that time, no conclusion was reached regarding participation in jazz dancing, fencing, ice skating, roller skating or in-line skating, rowing, speed 
Table 2. Classification of Sports Based on Level of Impact

\begin{tabular}{|c|c|c|c|}
\hline Low Impact & Potentially Low Impact & Intermediate Impact & High Impact \\
\hline \hline Stationary cycling & Bowling & Free weights & Baseball, softball \\
\hline Calisthenics & Fencing & Hiking & Volleyball \\
\hline Golf & Rowing & Horseback riding & American football \\
\hline Stationary skiing & Isokinetic weights & Ice skating & Racquetball, handball \\
\hline Swimming & Sailing & Lock climbing & Jogging, running \\
\hline Walking & Speed walking & Tennis & Lacrosse \\
\hline Ballroom dancing & Cross-country skiing & In-line skating & Doccer \\
\hline Water aerobics & Table tennis & Downhill skiing & Water-skiing \\
\hline
\end{tabular}

walking, downhill skiing, stationary skiing, weight lifting, and weight machines. Every other sport in the survey was either not recommended or had inconclusive data to make a recommendation. This classification system was taken one step further by Clifford and Mallon when they classified sports based on their level of impact and then made general recommendations for each different impact level, as seen in Table 2 [42].

As expected, Clifford and Mallon's recommendations allowed low-impact and potentially low-impact activities for most patients. In spite of their classification as low-impact, the possibility remains that participation in these sports may increase implant wear rate. Sports falling into the intermediate-impact category were recommended only for select patients. Patients participating in these activities should have a pre-activity evaluation, be closely monitored during involvement, and have specific guidelines for extent of involvement. High-impact sports were not recommended because the chance for injury and dislocation are increased, as is the probability of revision surgery. In 2007, Klein and associates published the consensus recommendations on return to athletic activity from the Hip Society and the American Association for Hip and Knee Surgeons [43]. A total of 614 surgeons responded to the survey, making it the largest performed to date. The questionnaire asked surgeons to consider 37 sporting activities and give advice as to whether the activities were allowed, allowed with experience, not allowed, or undecided. In this study, surgeons were asked to make recommendations based on the standard metal on polyethylene THA implant. The results of the survey can be seen in Table 3 .

An additional aspect of the survey was to assess the amount of time required to return to sports post-operatively. Surgeons varied in their responses, and 32\% allowed return in 1-3 months while 59\% required 3-6 months to elapse prior to returning to athletic activities.

A recent update of recommendations by the American Association for Hip and Knee Surgeons has been published [44]. This survey was limited to fifteen activities and had 139 responders. A consensus opinion was reached in allowing low-impact activities like walking on level surfaces, bicycling on level surfaces, climbing stairs, swimming, and golf. Also, high-impact activities such as jogging, sprinting, and skiing on difficult terrain were consistently discouraged. Singles tennis following THA was discouraged, but occasional doubles tennis (up to twice per month) was allowed. It was noted that surgeons with a higher operative volume were more liberal in activity recommendations and allowed more frequent athletic endeavors. This aspect was unique to the study in that previous surveys have not taken frequency of activity into consideration. It is well known that prosthetic wear is related to the number of load cycles, and therefore making recommendations based on frequency of activity seems advisable.

\section{THE ROLE OF REHABILITATION}

The role of rehabilitation has been discussed in relation to returning to sports after hip arthroplasty. It has been suggested that more intensive physical therapy postoperatively may prove beneficial to patients hoping to resume athletic activities [45]. Specifically, regimens aimed at improved hip abductor strength may improve the ability to return to sports. Also, it has been suggested that prolonged use of ambulatory assistive devices could actually improve functional outcomes in active patients [46]. This is in contrast to previous beliefs that progression to full weight bearing as rapidly as possible was best for outcomes relating to patient activity. In younger and more active patients, allowing for a slower return to full weight bearing may prevent patients from attempting higher impact activities too early in the post-operative period, thereby leading to better long-term functionality of the implant.

\section{CONCLUSIONS}

Due to the lack of long-term, prospective studies, making evidence based recommendations to patients regarding return to sporting activities following THA is difficult. However, the authors have used the available data to establish several key points regarding return to athletics. First, it is important to assess the patient's pre-operative athletic activity level and their competence in that area. Evaluation and documentation of both patient and surgeon expectations should be made in regard to post-operative sports participation. Any discrepancies should be discussed and resolved prior to the 
Table 3. Classification of Sports Based on Recommended Activity after THA

\begin{tabular}{|c|c|c|}
\hline Allowed Athletic Activity & Allowed with Experience & Not Allowed or Undecided \\
\hline \hline Golf & Downhill skiing & Squash \\
\hline Swimming & Cross-country & Jogging \\
\hline Doubles tennis & skiing & All contact \\
\hline StairMaster, elliptical & Weightlifting & Fports \\
\hline walking, treadmill & Ice skating & Sootball \\
\hline Speed walking & Rollerblading & Basketball \\
\hline Hiking & Pilates & High-impact \\
\hline Stationary skiing & & aerobics \\
\hline Bowling & & Baseball \\
\hline Road and stationary & & Softball \\
\hline cycling & & Snowboarding \\
\hline Low-impact aerobics & & Martial arts \\
\hline Rowing & & Singles tennis \\
\hline Dancing & & \\
\hline Weight machines & & \\
\hline
\end{tabular}

operation. Even patients with severe athletic or day-to-day limitations can likely expect a return to some level of their previous activity. Patients should all be given full disclosure that no mid-term or long-term data exist that support the return to athletics following THA, and thus their implant life may be adversely affected. During the operation, utilization of a minimally invasive posterior approach to the hip joint with repair of the short external rotators anecdotally appears to confer greater stability to the implant in the early postoperative period. In addition, surgeons should avoid violation of the abductors and should consider alternative bearing couples that have improved wear characteristics in active patients. Also, stability of the hip after implant placement is critical to reduce the risk of post-operative dislocation. These surgical considerations help to maximize the stability of the implanted hip joint and may be beneficial in rehabilitative efforts. Finally, routine post-operative radiographs should be performed yearly while the patient is participating in sports. This evaluation may allow the surgeon to detect early adverse changes with regard to the implant and alter patient activity level as needed. By keeping these and other factors in mind, orthopaedic surgeons performing THA can develop a patient appropriate plan for post-operative athletic activity and may improve long-term patient satisfaction with the implant.

\section{REFERENCES}

[1] Kurtz S, Ong K, Lau E, Mowat F, Halpern M. Projections of primary and revision hip and knee arthroplasty in the United States from 2005 to 2030. J Bone Joint Surg Am 2007; 89: 780-5.

[2] Jackson DW. Total joint arthroplasty poised to see incredible growth in the coming decades. Orthop Today 2007; $27: 7$.

[3] Hoagland FT, Steinbach LS. Primary osteoarthritis of the hip: Etiology and epidemiology. J Am Acad Orthop Surg 2001; 9(5): 320-7.

[4] Hoaglund FT, Oishi CS, Gialamas GG. Extreme variations in racial rates of total hip arthroplasty for primary coxarthrosis: a population based study in San Francisco. Ann Rheum Dis 1995; 54(2): 107-10.
[5] Chitnavis J, Sinsheimer JS, Clipsham K, et al. Genetic influences in end-stage osteoarthritis: Sibling risks of hip and knee replacement for idiopathic osteoarthritis. J Bone Joint Surg 1997; 79(4): 660-4.

[6] MacGregor AJ, Antoniades L, Matson M, et al. The genetic contribution to radiographic hip osteoarthritis in women: results of a classic twin study. Arthritis Rheum 2000; 43(11): 2410-6.

[7] Marti B, Knobloch M, Tschopp A, et al. Is excessive running predictive of degenerative hip disease? Controlled study of former elite athletes. BMJ 1989; 299(6691): 91-3.

[8] Khan NQ, Woolson ST. Referral patterns of hip pain in patients undergoing total hip replacement. Orthopedics 1998; 21(2): 123-6.

[9] Gill SD, McBurney H, Schulz DL. Land-based versus pool-based exercise for people awaiting joint replacement surgery of the hip or knee: results of a randomized controlled trial. Arch Phys Med Rehabil 2009; 90(3): 388-94.

[10] Noble PC, Conditt MA, Cook KF, Mathis KB. The John Insall Award: Patient expectations affect satisfaction with total knee arthroplasty. Clin Orthop Relat Res 2006; 452: 35-43.

[11] Massin P, Geais L, Astoin E, et al. The anatomic basis for the concept of lateralized femoral stems: A frontal plane radiographic study of the proximal femur. J Arthroplasty 2000; 15(1): 93-101.

[12] Reikeras O, Bjerkreim I, Kolbenstvedt A. Anteversion of the acetabulum and femoral neck in normals and in patients with osteoarthritis of the hip. Acta Orthop Scand 1983; 54(1): 18-23.

[13] Feugier P, Fessy MH, Bejui J, Bouchet A. Acetabular anatomy and the relationship with pelvic vascular structures: Implications in hip surgery. Surg Radiol Anat 1997; 19(2): 85-90.

[14] Roach KE, Miles TP. Normal hip and knee active range of motion: The relationship to age. Phys Ther 1991; 71(9): 656-65.

[15] Berend KR, Lombardi AV Jr, Welch M, Adams JB. A constrained device with increased range of motion prevents early dislocation. Clin Orthop Relat Res 2006; 447: 70-5.

[16] McGrory BJ, Morrey BF, Cahalan TD, et al. Effect of femoral offset on range of motion and abductor muscle strength after total hip arthroplasty. J Bone Joint Surg Br 1995; 77(6): 865-9.

[17] Walker PS. Design and Performance of joint replacements. In Chapman's Orthopedic Surgery. $3^{\text {rd }}$ ed. Philadelphia, PA: Lippincott, Williams, \& Wilkins 2000; vol. 3.

[18] Woo RY, Morrey BF. Dislocations after total hip arthroplasty. J Bone Joint Surg Am 1982; 64(9): 1295-306.

[19] White RE Jr, Forness TJ, Allman JK, Junick DW. Effect of posterior capsular repair on early dislocation in primary total hip replacement. Clin Orthop Relat Res 2001; 393: 163-7. 
[20] Pellici PM, Bostrom M, Poss R. Posterior approach to total hip replacement using enhanced posterior soft tissue repair. Clin Orthop Relat Res 1998; 355: 224-8.

[21] Masonis JL, Bourne RB. Surgical approach, abductor function, and total hip arthroplasty dislocation. Clin Orthop Relat Res 2002; 405: 46-53.

[22] Witzleb WC, Stephan L, Krummenauer F, Neuke A, Günther KP. Short-term outcome after posterior versus lateral surgical approach for total hip arthroplasty - A randomized clinical trial. Eur J Med Res 2009; 14(6): 256-63.

[23] Berger RA. Total hip arthroplasty using the minimally invasive two-incision approach. Clin Orthop Relat Res 2003; 417: 232-41.

[24] Dorr LD, Wan Z, Gruen T. Functional results in total hip replacement in patients 65 years and older. Clin Orthop Relat Res 1997; 336: 143-51.

[25] Engh CA, Hopper RH Jr, Engh CA Jr. Long-term porous-coated cup survivorship using spikes, screws, and press-fitting for initial fixation. J Arthroplasty 2004; 19 (7 Suppl 2): 54-60.

[26] Callaghan JJ, Templeton JE, Liu SS, et al. Results of Charnley total hip arthroplasty at a minimum of thirty years: A concise follow-up of a previous report. J Bone Joint Surg Am 2004; 86(4): 690-5.

[27] Keener JD, Callaghan JJ, Goetz DD, et al. Twenty-five-year results after Charnley total hip arthroplasty in patients less than fifty years old: A concise follow-up of a previous report. J Bone Joint Surg Am 2003; 85(6): 1066-72.

[28] Liu F, Jin Z, Roberts P, Grigoris P. Importance of head diameter, clearance, and cup wall thickness in elastohydrodynamic lubrication analysis of metal-on-metal hip resurfacing prostheses. Proc Inst Mech Eng [H] 2006; 220(6): 695-704.

[29] D'Antonio J, Capello W, Manley M. Alumina ceramic bearings for total hip arthroplasty. Orthopedics 2003; 26(1): 39-46.

[30] Silva M, Lee KH, Heisel C, et al. The biomechanical results of total hip resurfacing arthroplasty. J Bone Joint Surg Am 2004; 86(1): 40-6.

[31] Nishii T, Sugano N, Miki H, et al. Five-year results of metal-onmetal resurfacing arthroplasty in Asian patients. J Arthroplasty 2007; 22(2): 176-83

[32] Narvani AA, Tsiridis E, Nwaboku HC, Bajekal RA. Sporting activity following Birmingham hip resurfacing. Int J Sports Med 2006; 27(6): 505-7.
[33] Naal FD, Maffiuletti NA, Munzinger U, Hersche O. Sports after hip resurfacing arthroplasty. Am J Sports Med 2007; 35(5): 705-11.

[34] Wylde V, Blom A, Dieppe P, Hewlett S, Learmonth I. Return to sport after joint replacement. J Bone Joint Surg Br 2008; 90(7): 920-3.

[35] Visuri T, Honkanen R. Total hip replacement: its influence on spontaneous recreation habits. Arch Phys med Rehabil 1980; 61(7): 325-8.

[36] Dubs L, Gschwend N, Munzinger U. Sport after total hip arthroplasty. Arch Orthop Trauma Surg 1983; 101(3): 161-9.

[37] Ritter MA, Meding JB. Total hip arthroplasty. Can the patient play sports again? Orthopedics 1987; 10(10): 1447-52.

[38] Kilgus DJ, Dorey FJ, Finerman GA, Amstutz HC. Patient activity, sports participation, and impact loading on the durability of cemented total hip replacements. Clin Orthop Relat Res 1991; 269: 25-31.

[39] Huch K, Müller KA, Stürmer T, Brenner H, Puhl W, Günther KP Sports activities 5 years after total knee or hip arthroplasty: the Ulm Osteoarthritis Study. Ann Rheum Dis. 2005; 64: 1715-20.

[40] Healy WL, Iorio R, Lemos MJ. Athletic activity after joint replacement. Am J Sports Med 2001; 29(3): 377-88.

[41] McGrory BJ, Stuart MJ, Sim FH. Participation in sports after hip and knee arthroplasty: review of literature and survey of surgeon preferences. Mayo Clin Proc 1995; 70: 342-8.

[42] Clifford PE, Mallon WJ. Sports after total joint replacement. Clin Sports Med 2005; 24(1): 175-186.

[43] Klein GR, Levine BR, Hozack WF, et al. Return to athletic activity after total hip arthroplasty consensus guidelines based on a survey of the Hip Society and American Association of Hip and Knee Surgeons. J Arthroplasty 2007; 22(2): 171-5.

[44] Swanson EA, Schmalzried TP, Dorey FJ. Activity recommendations after total hip and knee arthroplasty: a survey of the American Association for Hip and Knee Surgeons. J Arthroplasty 2009; 24(6 Suppl): 120-6.

[45] Jacobs CA, Christensen CP, Berend ME. Sport activity after total hip arthroplasty: Changes in surgical technique, implant design, and rehabilitation. J Sport Rehabil 2009; 18(1): 47-59.

[46] Perrin T, Dorr LD, Perry J, Gronley J, Hull DB. Functional evaluation of total hip arthroplasty with five- to ten-year follow-up evaluation. Clin Orthop Relat Res 1985; (195): 252-60.

(C) Ross and Brown; Licensee Bentham Open.

This is an open access article licensed under the terms of the Creative Commons Attribution Non-Commercial License (http://creativecommons.org/licenses/by-nc/3.0/) which permits unrestricted, non-commercial use, distribution and reproduction in any medium, provided the work is properly cited. 